\title{
PERSPEKTIVEN DER PHILOSOPHIE
}

\author{
Neues Jahrbuch
}

Herausgegeben von

Rudolph Berlinger - Eugen Fink †

Friedrich Kaulbach - Wiebke Schrader

Band $7-1981$

Gerstenberg Verlag

Hildesheim
Rodopi N. V.

Amsterdam 


\section{Die Intention des Jahrbuches}

Die Perspektiven der Philosophie eröffnen Forschern, die die Arbeit philosophischer Begründung und Rechtfertigung des Denkens auf sich nehmen, eine Publikationsmöglichkeit.

Das Jahrbuch begreift sich nicht als Schulorgan einer philosophischen Lehrmeinung. Kontroversen sollen ausgetragen werden, wenn sich dadurch Probleme konturieren und Argumente verschärfen. Das Jahrbuch sieht seine Aufgabe darin, an der Intensivierung eines wissenschaftlichen Philosophierens mitzuarbeiten.

Die Jahrbuchbände bringen jeweils Beiträge zu einem Rahmenthema und solche nach freier Wahl. Rezensionen und Informationen zur philosophischen Diskussion sind vorgesehen.

Herausgeber und Redaktion

(C) Gerstenberg Verlag, Hildesheim 1981

Satz: Verlag Königshausen/Neumann, Würzburg

Druck: Strauss \& Cramer, 6945 Hirschberg II Verarbeitung: Karl Schaumann, 6100 Darmstadt

ISSN 3-8067-0171-1288

Best. Nr. 3-8067-1290 


\section{Hans Peter Balmer}

\section{NIETZSCHES ERSCHLIESSUNG DER EUROPÄISCHEN MORALISTIK}

Mit den folgenden Darlegungen soll die These aufgestellt und mittels einiger knapper Ausführungen belegt werden, daß Friedrich Nietzsche einer philosophischen Erschließung der europäischen Moralistik die wesentlichen Impulse gegeben hat. Unter Moralistik ist ein kontinuierlicher Strom anthropologischer Reflexion zu verstehen, der sich, aus antiken Quellen entspringend, von Italien, Frankreich, Spanien, England und Deutschland als unsystematische philosophisch-literarische Breviloquenz über die verschiedenen europäischen ${ }^{1}$ Kulturkreise fortbewegt, immer an die individuelle Freiheit appellierend. Eine Zusammenstellung und Aufarbeitung der moralistischen Überlieferung - bislang nur in Ansätzen von August Buck, Hugo Friedrich, Gerhard Hess, Margot Kruse, Gerhard Neumann, Corrado Rosso ${ }^{2}$, Fritz Schalk, Gerhart Schröder und Claus Uhlig angebahnt - ergibt eine Philosophie der menschlichen Dinge ${ }^{3}$.

Die menschlichen Dinge (res humanae, le cose umane, les choses humaines, las cosas humanas, things amongst man) bezeichnen - seit die Griechen sie als pragmata, nicht aber als chrēmata oder gar hypokeimena zu denken begannen - den Um-

1. Zur Kennzeichnung der Moralistik als europäisches Phänomen vgl. beispielsweise I moralisti classici, Da Machiavelli a La Bruyère, hrsg. v. G. Macchia, Mailand 1961.

2. Eine zusammenfassende Darstellung der Forschung des italienischen Gelehrten wäre sehr zu wünschen; vgl. insbes.: Figure e dottrina della filosofia dei valori, Turin 1949; Moralisti del „bonheur“, Turin 1954; Virtù e critica della virtù nei moralisti francesi, Turin 1964; La „Maxime“", Saggi per una tipologia critica, Neapel 1968; Montesquieu moraliste, Bordeaux 1971; Rassegna bibliografica sui moralisti francesi, in: Rivista di letterature moderne e comparate 25 (1972), S. 269-302.

3. Näheres' zur europäischen Moralistik bei H. P. Balmer, Philosophie der menschlichen Dinge, Bern/München: Francke 1981. 
kreis menschlicher Praxis schlechthin, den Inbegriff alles dessen, womit wir es als Menschen immer schon zu tun haben: keine Gegenstände, nichts Festes, Abgeschlossenes, sondern als Grundstruktur gelebten Lebens ingesamt die Ungegenständlichkeit und Unverfügbarkeit unseres Daseins in der Welt. Die Philosophie der menschlichen Dinge ist auf Behebung von Vergegenständlichung, Segregation, Disqualifikation und Exkommunikation jeder Art aus; ihr Anliegen ist Integration, Teilhabe am unverkürzten Leben. Sie gewinnt und behält ihre Bedeutung aus dem Respekt vor der unabsehbaren Vielfalt des Menschlichen und aus dem persönlichen Beteiligtsein jedes Menschen, der sie aufgreift. Verstehen gibt es nur approximativ, bedarf der Beziehung und der empathisch-teilnehmenden Einfühlung. Die letzte Instanz bleibt der einzelne Mensch. Durch Verständnis, Entscheidungshilfe und Rat kann seine Selbstvertretung im günstigen Fall unterstützt, niemals aber ersetzt werden. Moralische Normen - das hat die Psychoanalyse zuletzt geradezu gesetzmäßig erhärtet -, sofern sie den Menschen manipulieren wollen und seine Freiheit außer acht lassen, rufen zwangsläufig Widerstand hervor. Die Verhärtung durch die moralischen Machenschaften wird von den Moralisten wieder aufgebrochen: Sie stellen ihre Beobachtungen und Reflexionen der freien Begutachtung anheim; ex ingenio suo quisque demat vel addat fidem ${ }^{4}$ sagt der moralistische Historiker Tacitus, dem in seiner conscientia generis humani ${ }^{5}$ so viel von den Bedingungen menschlichen Zusammenlebens und den Abgründen der Macht aufgegangen war. Die Sentenzen der Moralisten sind keine Dogmen. Das letzte Wort behält jederzeit der angesprochene Mensch.

Den „Überblick über die Bedürfnisse der Menschheit" ${ }^{* 6}$, den die Moralistik ermöglicht, ist niemals der Versuch der Etablierung fester Werte, sondern vielmehr Differenzierung der Frage nach dem Menschen im Aufzeigen von Entscheidungsspielräumen: Das

4. Tacitus, Germania, 3.

5. Ders., Aricola, 2,2.

6. F. Nietzsche, Menschliches, Allzumenschliches, I, 25: S I, 466; Nietzsche wird im folgenden stets abgekürzt zitiert: Sigel für das Werk, römische 
Wissen darum, wie die Menschen leben, um sie zu verstehen und aus der Orientierung darüber, wie man leben kann, Vergleich und Besinnung zu ermöglichen und vielleicht einen Anhalt für die Entscheidung, wie wir leben wollen, zu gewinnen.

Was von den Moralisten befürwortet wird, ist ein Ethos der Freiheit, es läßt sich aus keiner Prämisse stringent deduzieren, sondern nur von Menschen als universale Versöhnung praktisch vollziehen.

I.

Nach dieser kurzen Vorverständigung ist zunächst einiges anzudeuten zu Nietzsches Stellung innerhalb der nachidealistischen Philosophie, dann zur Bedeutung der Moralistik im corpus Nietzscheanum. Es kommt dabei darauf an, das Bild, das Nietzsche von dem moralistischen Überlieferungsstrang hatte, $\mathrm{zu}$ skizzieren und weiterhin auf die Bedeutung hinzuweisen, die er einer Erschließung dieser Tradition zuwies.

Die Auflehnung gegen die Vollendung der Metaphysik im deutschen Idealismus ist - der Motivation wie der Ausfuhrung nach - ein wesentlich anthropologisches Geschehen. Wird schon in Fichtes Bestimmung des Triebes eine erste Kurskorrektur erkennbar, so geht beim späten Schelling die Vernunft ihrer absoluten Position zu Gunsten von Wollen, Erfahrung, Wirklichkeit, Persönlichkeit und Leben endgültig verlustig. Der Bruch mit der Spekulation erlaubt Feuerbach eine Rehabilitierung von Sinnlichkeit und Sozialität, während Stirner und Kierkegaard die Einzelexistenz zum Ausgang eines antiidealistischen Philosophierens machen.

In allen diesen verschiedenen Positionen sind jedoch unschwer wiederum Absolutsetzungen zu erkennen. Dieses eher unbewußte, quasi-metaphysische Begründen der neuen Erfahrung von Welt

Zahl für Buch, arabische Zahl für Kapitel, Abschnitt, daraufhin nach Doppelpunkt der Verweis auf Band (römische Zahl) und Seite (arabische Zahl) der Ausgabe von K. Schlechta; Näheres zur Zitationsweise bei H. P. Balmer, Freiheit statt Teleologie, Ein Grundgedanke von Nietzsche, Freiburg/München 1977 (Symposion, 55), S. 8, 127. 
und Mensch trifft - trotz der Vorordnung von Anschauung und Erfahrung - auch auf den Schopenhauerschen Dualismus von Wille und Vorstellung zu. Selbst Nietzsche, der am entschiedensten von der Metaphysik loskommt, gerät, wo er sich anschickt, ein höheres Menschentum vorzuzeichnen, doch wieder - diesmal in der Weise einer immanenten anthropologischen Teleologie - unter ihren Bann. Seine Philosophie weist, aller Befreiung von der Metaphysik zum Trotz, noch einen moralisch-normativen Zug auf: Sie erblickt in der Erhöhung des Menschen das Ziel der menschlichen Entwicklung. Vor dem Telos der Erhebung zum Transhumanen sinken alle menschlichen Wirklichkeiten zu bloßen Vorstufen herab.

Im Gegensatz zu dieser durchgängigen Verflechtung von metaphysischer Lösung und Verhaftung begegnet uns in Nietzsches Werk eine realistische Tendenz, der an der deskriptiven Erfassung des wirklichen Menschentums in der ganzen Breite seiner Erscheinungen und Möglichkeiten gelegen ist. Es ist dieser moralistische Zug in Nietzsches Denken, dem wir uns in unserer Situation mit besonderem Gewinn zuwenden.

Nietzsches Erschließung der moralistischen Überlieferung ist bereits durch Kant und Schopenhauer vorbereitet. Kants „Humanistik" treffend benennt - findet sich vorzugsweise in vier Schriften: in den „Beobachtungen über das Gefühl des Schönen und Erhabenen" aus der vorkritischen Periode, dann im Dritten Abschnitt „Von der Macht des Gemüts, durch den bloßen Vorsatz seiner krankhaften Gefühle Meister zu sein" aus dem „Streit der Fakultäten", weiterhin in der ,Anthropologie in pragmatischer Hinsicht" und schließlich in der ,Pädagogik" aus der nachkritischen Phase. Wird - wie bei Friedrich Kaulbach - die Leistung der pragmatischen Vernunft als ,eine ständige Umorientierung und Entlarvung“ 8 bezeichnet, so ist damit die sachliche Nähe zur mora-

7. I. Kant, Der Streit der Fakultäten, in: Akademie-Ausgabe, Bd. VII, S. 28.

8. F. Kaulbach, Weltorientierung, Weltkenntnis und pragmatische Vernunft bei Kant, in: ders., Kritik und Metaphysik, Berlin 1966, S. 75. 
listischen Uberlieferung aufgewiesen. Indessen ist die Integration rhapsodischer Moralistik bei Kant von einer eigentümlichen Problematik infolge des Übergewichts an englischem Einfluß und der Hintansetzung der großen Klassiker des Genus wie beispielsweise Montaigne hinter moralisch-moralistisch ambivalenten Denkern wie Descartes, Pascal, Fénelon und Rousseau. Das Ungenügende, bisweilen Hilflose und nicht selten Lebensfremde bei Kant sieht nicht erst Nietzsche, sondern bereits Lichtenberg, wenn er in seinem unerreichten, liebenswürdig sublimen Witz mutmaßt: „Sollte nicht manches von dem was Herr Kant lehrt, zumal in Rücksicht auf das Sittengesetz Folge des Alters seyn, wo Leidenschaft und Neigung ihre Krafft verloren haben, und Vernunfft allein übrig bleibt?" 9

Ein moralistisches Konzept von Philosophie entwickelt, ohne ihn jedoch konsequent treu zu bleiben, Arthur Schopenhauer. Im Unterschied zur Transzendentalphilosophie Kants und zum deutschen Idealismus bedeutet Philosophie für Schopenhauer Erfassen, nicht Hervorbringen von Wirklichkeit, Auslegung von Erfahrung, nicht Erklärung der Welt ausletzten Gründen: Philosophie bleibt „,bei dem Tatsächlichen der äußern und innern Erfahrungen, wie sie jedem zugänglich sind, stehn und weist den wahren und tiefsten Zusammenhang derselben nach, ohne jedoch eigentlich darüber hinauszugehn $z u$ irgend außerweltlichen Dingen und deren Verhältnissen zur Welt. Sie macht demnach keine Schlüsse auf das jenseit aller möglichen Erfahrung Vorhandene, sondern liefert bloß die Auslegung des in der Außenwelt und dem Selbstbewußtsein Gegebenen, begnügt sich also damit, das Wesen der Welt seinem innern Zusammenhange mit sich selbst nach zu begreifen" 610 . Philosophie erbringt Einsicht, die Schopenhauer auf Erfahrung und Beobachtung gründet und als deren Ursprung er, ähnlich wie Herder, die anschauliche Erkenntnis durch den Menschen ausmacht.

9. G. C. Lichtenberg, hrsg. v. A. Leitzmann, L 733.

10. A. Schopenhauer, Sämtliche Werke, hrsg. v. W. v. Löhneysen, 5 Bde., Frankfurt am Main 1960-65, Bd. II, S. 821. 
Wie bei Vauvenargues und Lichtenberg hat deswegen der Mensch als ganzer und ungeteilter am Ende dazustehen und zur Aktion zu kommen: „Eine seltsame und unwürdige Definition der Philosophie, die aber sogar noch Kant gibt, ist diese, daß sie eine Wissenschaft aus bloßen Begriffen wäre. Ist doch das ganze Eigentum der Begriffe nichts anderes, als was darin niedergelegt worden, nachdem man es der anschaulichen Erkenntnis abgeborgt und abgebettelt hatte, dieser wirklichen und unerschöpflichen Quelle aller Einsicht. Daher läßt eine wahre Philosophie sich nicht herausspinnen aus bloßen abstrakten Begriffen, sowohl innere als äußere. Auch nicht durch Kombinationsversuche mit Begriffen, wie sie oft ... ausgeführt worden sind, wird je etwas Rechtes in der Philosophie geleistet werden. Sie muß so gut wie Kunst und Poesie ihre Quelle in der anschaulichen Auffassung der Welt haben: auch darf es dabei, so sehr auch der Kopf oben zu bleiben hat, doch nicht so kaltblütig hergehn, daß nicht am Ende der ganze Mensch mit Herz und Kopf zur Aktion käme und durch und durch erschüttert würde. Philosophie ist kein Algebra-Exempel. Vielmehr hat Vauvenargues gesagt: ,Les grandes pensées viennent du çœur “ “11.

Während der anthropologische Wirklichkeitssinn bei Kant und Schopenhauer eher beiläufig zum Tragen kommt und immer wieder von Schemata, idealistischer Systematik und teleologischer Deutung überfrachtet wird, geht Nietzsche weit darüber hinaus. Er erfaßt als erster die europäische Moralistik in ihrem Zusammenhang, ihrer Gliederung und ihrer Bedeutung. In Verbindung mit dem Verzicht auf Teleologie gewinnt der Strang deskriptiv-anthropologischer Brachylogie den Stellenwert eines naturalistischen Gegengewichts gegen eine idealistisch-final konzipierte Einheitsgeschichte. Im Moment der Falsifizierung des metaphysischen Geschichtskonzepts im historischen Bewußtsein wirkt Nietzsches praktische Philosophie dem totalen Versinken alles geschichtlich Konkreten ins Nichts machtvoll entgegen. In der Überwindung des Nihilismus, der infolge der Entwertung der Werte Platz greift, erlangt die Moralistik schließlich welthistorische Geltung. 
Wie Horneffer, Andler und Krökel schon nachgewiesen haben, bereitet sich bei den Moralisten jene Selbstbesinnung Europas vor, die schließlich in Nietzsche kulminiert ${ }^{12}$. Dem deutschen Philosophen des 19. Jahrhunderts gelten die Werke der Moralisten als wahrhaft „europäische Bücher" (MA/WS 214). Die Aphorismen, die sie enthalten, bedürfen intensiver und kunstgerechter Interpretation. Eine Grundregel dieser Hermeneutik verlangt die strikte Enthaltung von philosophischer Verallgemeinerung. Bis zu Schopenhauer haben die Philosophen ,die Sätze der Menschenprüfer“, der „Moralisten“, wie Nietzsche in Klammern erläutert (MA II, 5), unbedingt genommen und damit verdorben. Auf deutscher Seite läßt Nietzsche einzig die Aphorismen Lichtenbergs und die „Psychologischen Beobachtungen“, die Paul Rée 1875 herausbringt, als lesenswerte Moralistik gelten.

Vor dem Hintergrund der moralistischen Überlieferung, die Nietzsche „mehr wirkliche Gedanken als alle Bücher deutscher Philosophen zusammengenommen" (MA II/WS 214) zu enthalten scheint, nehmen sich neben Schopenhauer auch Kant und Schiller und zunächst sogar Goethe defizitär aus. Später jedoch in der „Götzen-Dämmerung“ würdigt Nietzsche Goethe als ein europäisches Ereignis: „Was er wollte, das war Totalität; er bekämpfte das Auseinander von Vernunft, Sinnlichkeit, Gefühl, Wille"; Goethe, sagt Nietzsche nunmehr, versuchte, zur „Natürlichkeit der Renaissance" hinaufzugelangen und konzipierte demgemäß einen Menschen, ,,der sich den ganzen Umfang und Reichtum der Natürlichkeit zu gönnen wagen darf". Was Nietzsche in diesem Zusammenhang als Grundintention Goethes hervorhebt, verdeutlicht die Bestrebungen der Moralistik insgesamt: „,eine Universalität im Verstehn, im Gutheißen, ein An-sich-herankommen-lassen von jedwedem, einen verwegnen Realismus, eine Ehrfurcht vor allem Tatsächlichen" (GD IX, 49f). In Goethe findet Nietzsche die Vor-

12. A. Horneffer, Nietzsche als Moralist und Schriftsteller, Jena 1906; C. Andler, Nietzsche, Bd. I, Les précurseurs de Nietzsche, Paris 1920, 3. Aufl. 1958; E. Krökel, Europas Selbstbesinnung durch Nietzsche, Ihre Vorbereitung bei den französischen Moralisten, München 1929. 
zeichnung einer europäischen Kultur, die ,die volle Erbschaft der schon erreichten Humanität macht" (WM 104).

Die moralistische Welt- und Menschenkenntnis wird für Nietzsche zum Prüfstein einer unterschiedlichen Wertung von deutschem Geist und französischem Geist. Jahrhunderte ,moralistischer Arbeit" und die Angewiesenheit Europas auf diese ,alte vielfache moralistische Kultur" ( $\mathrm{J} 254$ ) begründen die Überlegenheit der Franzosen. Über Chamfort, Vauvenargues, Fontenelle, La Bruyère, La Rochefoucauld und Montaigne gewinnt Nietzsche Verbindung zur Renaissance und weiter zurück zur römischen und griechischen Antike, dem Urbild ,aller moralistischen Freisinnigkeit" (MA II, 220).

Die „Kultur der unbefangensten Weltkenntnis“, die „Freude an allem Typischen des Menschen und der Ereignisse" (M 168) sieht Nietzsche noch in dem von ihm so genannten „MenschenDenker" Thukydides blühen, der ihm deswegen und wegen der Nähe zur Anthropologie der griechischen Sophistik gerechter und, zusammen mit den griechischen Mythen und Tragödien, weiser als die Sokratisch-Platonisch-Aristotelische Philosophie erscheint. Der Thukydideischen Gnomik konnte Nietzsche weiterhin die enge Verflochtenheit der Phänomene der Macht mit dem Wesen des Menschen (anthrōpeia phýsis) entnehmen ${ }^{13}$. Über das Inhaltliche hinaus stößt Thukydides bereits zu methodisch grundlegenden Einsichten in die Bedeutung der gnomischen Apperzeption vor: Gnome umfaßt in ursprünglicher Weise den Zusammenhang menschlichen Weltverhaltens, indem Absichten genannt und Handlungen auf Motive zurückgeführt werden; die Reflexion bietet die Möglichkeit, die Praxis im einzelnen einsichtig zu machen und insgesamt in Interdependenz mit den Bedingungen menschlicher Existenz zu sehen $^{14}$. Für die Grundsituation des Menschen verwendet Thukydides bereits einen festen Begriff: to anthrópinon, das Äquivalent zu

13. Vgl. E. Topitsch, Anthropeia phýsis und Ethik bei Thukydides, in: Wiener Studien 61/62 (1943-47), S. 50-67.

14. C. Schneider, Information und Absicht bei Thukydides, Untersuchung zur Motivation des Handelns, Göttingen 1974. 
dem, was seit Cicero als condicio humana den Horizont des humanistischen und dann, seit Montaigne, des spezifisch moralistischen Fragens nach dem Menschen und seinem Leben abgibt.

Die humanistische Vorstufe zur Moralistik während der Epoche der italienischen Renaissance mit ihren Nachwirkungen in Spanien und Deutschland scheint Nietzsche allenfalls summarisch gekannt zu haben. Weder Guiccardinis „Ricordi“, die der unablässigen Veränderlichkeit aller Verhältnisse (la variazione naturale delle cose del mondo) ${ }^{15}$ Rechnung zu tragen suchen, noch Girolamo Cardanos überaus enge Annäherung an die Denkweise der klassischen Moralisten greift Nietzsche auf. Die enge Verbindung seines Denkens zu Niccolò Machiavellis Doppelwerk „Discorsi“" und „Il principe“ mit ihrer Fülle pragmatischer Reflexionen wird nur in einer pauschalen Berufung auf den Begriff virtù, nicht aber auf dessen Rückbindung in die Termini necessità und occasione explizit.

Montaigne ist der einzige, den Nietzsche immer - und oft an der Spitze - anfuihrt, wenn er, mit sonst wechselnden Namen, das Spektrum der europäischen Moralistik ausleuchtet. Im Unterschied zu Hegel und Dilthey erkennt Nietzsche das Singuläre und Unzeitgemäße an Montaigne, der mitten in den Wirren der Reformation der Mehrung der „Lust auf dieser Erde zu leben“ (UB III, 2) hingegeben ist. Die überragende Stellung Montaignes sieht er insbesondere dadurch gefestigt, daß der größte nachantike Dramatiker, William Shakespeare, ,,sein bester Leser“ (UB IV , 3) und sein „Vollender" (UW I, 538) wird. Montaigne, das ist Nietzsche ein ,Höhepunkt" kosmologisch-anthropologischer "Redlichkeit" (UW II, 1077). Seiner Weisheit, die mit Platonismus so wenig zu schaffen hat wie die Weisheit Heraklits, Demokrits, Epikurs, Horazens oder später Camus', konnte Nietzsche neben dem ateleologischen Weltbild (un mouvement irregulier, perpetuel, sans patron, et sans but) ${ }^{16}$ den paradoxen Grundsatz moralistischer Anthropologie

15. F. Guicciardini, Ricordi, hrsg. v. R. Spongano, Florenz 1951, C 114. 16. M. de Montaigne, Oeuvres complètes, hrsg. v. A. Thibaudet/M. Rat, Paris 1962, III, xiii, S. 1045. 
entnehmen, wonach der ehrliche Zugang zum Allerindividuellsten zugleich die Entdeckung der Gesamtform des Menschseins mit sich bringt: Chaque homme porte la forme entière de l'humaine condition $^{17}$. Nicht im Streben nach an sich seienden, unvergänglichen, mit sich identischen Idealen, gipfelnd in einem höchsten Ziel, sondern im Bestehen des Hier und Jetzt liegt der Sinn. Am Gegenwärtigen erfährt sich der Geist als sinnlich, erfahren die Sinne sich als geistig: Intellectuellement sensibles, sensiblement intellectuels ${ }^{18}$. „Es gibt“, sagt Montaigne, „keine schwierigere Wissenschaft als dieses Leben recht und natürlich zu leben, und die schlimmste aller Krankheiten ist die, unser Dasein zu verachten "19.

Von ähnlich gewichtiger Bedeutung wie Montaigne ist für Nietzsche Nicolas Chamfort, der Zeitgenosse der Französischen Revolution, mit seiner Neigung zum Extrem und seiner glühenden Unbedingtheit. Bei ihm findet Nietzsche die Intention auf die „Heiligung des Lachens“, das Indiz für den erstrebten „Sieg über den Geist der Schwere" (UW II, 1376), des weiteren das Bild vom Hammer und die Devise ,hart werden“ (Z III). Hier trifft er auch auf die Gestalt des Diogenes, liest von ,christlichem Pöbel' und von Maulwurfs- und Adlerklugheit, sieht vor allem den Dualismus von Gut und Böse auf zoroastrisch-persische Theologie zurückgeführt. Hier ist ein Lob der solitären Existenz vorhanden und erscheint - in einer Tradition, die über Mark Aurel und Horaz bis zu Empedokles zurückführt - der Kreis ${ }^{20}$ als ein Bild jener umfänglichsten Seele, die zum Schluß des "Zarathustra" von der großen Sehnsucht zu singen anhebt.

In einem Atemzug mit Chamfort erwähnt und wie dieser mit

17. ebd., III, ii, S. 782.

18. ebd., III, xiii, S. 1087.

19. „Il n'est ... ny science si ardue que de bien et naturellement scavoir vivre cette vie; et de nos maladies la plus sauvage, c'est mespriser nostre estre“" (ebd. S. 1091).

20. Zur Vorgeschichte der Kreis-Metapher bis zurück zum sphairós des frühgriechischen Denkers Emmpedokles vgl. R. List-Marzolff, Sébastien-Roche Nicolas Chamfort, München 1966, S. 148. 
größter Anerkennung bedacht werden von Nietzsche Stendhal und Galiani. In Stendhal sieht er den antizipierenden, Zeiten und Völker universal synthetisierenden „Ausspürer und Entdecker" ( $\mathrm{J}$ 254) der europäischen Seele. Einsamkeit und Narretei des zerbrochenen Herzens verehrt Nietzsche an Galiani, dessen tragisches Wissen ihn in Abstand zur Oberflächlichkeit der Aufklärung setzt. Vauvenargues, bei dem die Grenzen der Aufklärung mindestens ebenso deutlich sichtbar werden, dazu aus vitalistischer Sicht in vielen Belangen eine Umwertung vorgenommen, ein Dynamismus pluraler Kräfte konzipiert und bis in die Terminologie hinein die Philosophie des Willens zur Macht ${ }^{21}$ vorformuliert wird, gelangt, wohl aus mangelnder Kenntnis, nicht zu angemessener Würdigung. „Die Körperwelt“, so ist bei Vauvenargues zu lesen, ,hängt nicht von einem Prinzip (un premier principe) oder einer allumfassenden Ursache ab (une cause universelle), wie man glaubt. Denn ich, als freies Wesen, muß nur auf ein wenig Schnee hauchen, um das ganze Natursystem zu stören (je dérange tout le système de l'univers). Seltsame Täuschung! zu glauben, daß ein einziges Gesetz die ganze Natur beherrsche, während die Erde von hundert Millionen Kraftzentren beherrscht ist (la terre est couverte de cent mille millions de petits agents), die alle nach ihrer Laune diese Macht durchkreuzen" ${ }^{22}$.

Unter den englisch schreibenden Moralisten nimmt Emerson, insbesondere mit seinen Reflexionen zur Problematik der Macht, den größten Einfluß auf Nietzsche. Heiterkeit, Güte, Dankbarkeit, Zufriedenheit, Geist sind Züge, verwandt denen Montaignes, die Nietzsche an dem amerikanischen Essayisten hervorhebt. Demgegenüber tritt Pope in Nietzsches Werk an die Peripherie, und auch die utilitaristisch-zwielichtige Essayistik eines Francis Bacon vermag sich von dem generellen Verdikt Nietzsches über die englische Moralphilosophie nicht abzuheben.

21. Vgl. M. Wandruszka, Wille und Macht in drei Jahrhunderten französischer Schau, Stuttgart/Berlin 1942.

22. Vauvenarguks, Réflexions et Maximes, hrgs. v. S. de Sacy, Paris 1971, Aph. 595. 
Als Brücke zwischen der Vollendung bei den Franzosen des 16. und 17. Jahrhunderts und dem Fundament der Moralistik bei den frühen Griechen - den Anteil der biblischen und altorientalischen Weisheitsliteratur übersieht Nietzsche im Gegensatz zu Montaigne, Lichtenberg und Schopenhauer - wirken die hellenistischen und römischen Moralphilosophen, insbesondere Plutarch, Epikur, Seneca, Epiktet und Mark Aurel. In allen diesen Fällen ist Nietzsches Verschränkung komplex und der tatsächliche Einfluß nicht leicht zu ermitteln. Sokrates, Platon, Gracián, Schopenhauer sind ähnlich gelagerte Fälle, vor allem aber jene zwei Franzosen, die Nietzsche mit Abstand am häufigsten erwähnt: Pascal und La Rochefoucauld. Sie alle rechnet er jenem Typus korrumpierter Moralistik zu, der insgesamt Ausdruck der Verkümmerung des Menschen ist.

Nietzsches Beziehungen zu Gracián - zumindest durch Schopenhauer und über Chamfort hat er den spanischen Moralisten mit seiner unvergleichlichen arte de prudencia gekannt - bedürfen, über die Studie von Bouillier ${ }^{23}$ hinaus, weiterer Klärung. Festzuhalten ist: „Also sprach Zarathustra" steht als philosophische Dichtung auf einer Linie, die über Voltaires „Candide“ zum „Criticón“ zurückführt. Das Gleichnis vom Seiltänzer, ganz abgesehen vom Bild des tags mit der Laterne Menschen suchenden Diogenes, bringt, vor Chamfort und Galiani, Gracián. Gemeinsam sind außer ungezählten Übereinstimmungen in der moralistischen Anatomie (moral anatomia del hombre $)^{24}$ ein gewisser Heroismus, die Devise, hart wie Diamant zu werden, die auf Mark Aurel und Platon zurückgreifende Rede vom Seelchen, die Metaphern vom Schatten, vom Sand der Menschheit und von der als Schlange sich in den Schwanz beißenden, kreisförmigen Zeit, schließlich die Lehren von der ewigen Wiederkehr des Gleichen, von dem kaum zu überbrückenden Ge-

23. V. Bouillier, Baltasar Gracián et Nietzsche, in: Revue de littérature comparée VI (1926), S. 381-401.

24. Criticón I, ix; ín: Obras completas, hrsg. v. A. del Hoyo, Madrid 1960, S. 597. 
gensatz zwischen Wahrheit und Leben sowie von der Heilsamkeit, vieles nicht wissen zu wollen und mancherlei vergessen zu können.

„Das lehrreichste Opfer des Christentums“ (EH), wie er es versteht, ist für Nietzsche Pascal. An ihm wie an La Rochefoucauld, nicht jedoch an dem mindestens ebenso einschlägigen La Bruyère, liest er ab, wie teuer ,der melancholische Scharfsinn der Selbstverkleinerung" (WM 389) Europa zu stehen kommt. Pascals Syndrom ist in der Diagnose Nietzsches ein ,allgemeines Leiden der Modernen" (UW I, 46). Die mystische Negation in Pascals Apologetik, sein teleologisches Denken und der Selbsthaß bringen Nietzsche dazu, ihn in die umfangreiche ,,Literatur der Verleumdung des Lebens" (UW I, 547) einzureihen.

Im Zeichen ,christlicher Verdüsterung“ erscheint Nietzsche des weiteren La Rochefoucauld, dem er zwar nicht wie Pascal den radikalen Irrtum „Le moi est toujours haissable“ (W) aber doch eine Fehleinschätzung der Selbstwertgefühle zur Last legt. Während die Überzeugung vom Unwert des Mitleidens Anerkennung findet, wertet Nietzsche die Entlarvungskunst La Rochefoucaulds als et-

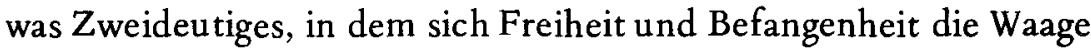
halten: Ein enttäuschter Idealist, so Nietzsches Urteil, setzt den Menschen herab und läßt im Grunde die herkömmliche Ordnung unangetastet. Der vermißte ,umgekehrte La Rochefoucauld“ (UW II, 507) kann indessen in weit höherem Maße als es Nietzsche bewußt war - dies hat die moderne Forschung ${ }^{25}$ gezeigt - in La Rochefoucauld selbst gefunden werden. Die traditionelle Vorstellung von den Verkehrtheiten im menschlichen Herzen steht ebenso wie die Rede von den christlichen Tugenden und das Insistieren auf Demut und Gesinnungsethik am Rand eines Denkens, das zentral nicht dem Laster die Tugend, sondern der Trägheit und Willensschwäche die Kraft (la force) und Festigkeit (la fermeté) entgegenstellt und hierin bereits ein Jenseits von Gut und Böse andeutet. Außer der subjektiven Empfindung (sensibilité) kennt La

25. Vgl. insbes. L. Ansmann, Die „Maximen“ von La Rochefoucauld, München 1972. 
Rochefoucauld keinen objektiven Maßstab für den Grad der Betroffenheit durch Widerfahrnisse und deren Wertung als Gut oder Übel. Die eigentlichen Chancen des Menschlichen sieht der große Moralist des 17. Jahrhunderts in einer direkteren Verschränkung mit der affektischen Grundstruktur. Aufrichtig sein (être sincère), fraglos die tiefste Intention, das Ethos von La Rochefoucaulds Maximen, bringt uns - ganz und gar nicht intellektualistisch-voluntaristisch gemeint - das Glück, natürlich zu sein (être naturel).

II.

Nietzsches eigener Beitrag an die moralistische Aphoristik findet sich verstreut überall in seinem veröffentlichten und nachgelassenen Werk; seine Philosophie der ,menschlichen Dinge“ (PG) konzentriert sich in den Büchern ,Menschliches, Allzumenschliches", „Morgenröte“, „Die fröhliche Wissenschaft", ,Jenseits von Gut und Böse“ und in Teilen von „Also sprach Zarathustra“. Im ersten Band von „Menschliches, Allzumenschliches“ sind die „ersten und letzten Dinge" in Betracht gezogen; die im engeren Sinn moralistischen Beobachtungen und Reflexionen zur sozialen und individuellen Situation des Menschen, verstanden als Ansätze zu einer neuen Kultur der Gerechtigkeit, sind in allgemeine Überlegungen zu diesem Thema eingebettet; Antworten und Fragestellungen bisheriger Moral und Kunst finden ebenso wie die Rolle der Wissenschaften einläßliche Berücksichtigung. Gegenüber dieser ,Systematik', die freilich in engstem Zusammenhang mit der Moralistik steht, bringen der zweite Band von ,Menschliches, Allzumenschliches" wie auch die folgenden Bücher ,Morgenröte“ und „Die fröhliche Wissenschaft" in der typischen lockeren Anordnung „Vermisehte Meinungen und Sprüche".

Das allgemeine Anliegen, das „Menschliches, Allzumenschliches" im ganzen umspannt und in die folgenden Werke hineinwirkt, geht darauf, ,wieder gute Nachbarn der nächsten Dinge“ zu werden. Diese klar gegen Platon und die auf ihn folgende Metaphysik abgesetzte Intention schließt an der Basis des Menschlichen 
die Umwandlung der ,Leidenschaften der Menschheit allesamt in Freudenschaften" ein (MA/WS 37: S I, 897) ${ }^{26}$.

Nietzsches Ersetzung moralischer Imperative durch moralistisches Appellieren kündigt sich in der gewichtigen zweiten ,Unzeitgemäßen Betrachtung“ deutlich an. Das ,Wozu' der Existenz will er, ähnlich wie Lichtenberg, einzig von einer Besinnung auf die ,echten Bedürfnisse“ (UB II, 9f) gewinnen. Die Moralistik spricht „die Sprache der auch im Menschlichen wiederhergestellten Natur" (UB IV, 11); ihr Ziel ist eine menschliche Weisheit, ein neues, umfänglich-gerechtes Weltverhalten. Es gilt, den ,Zusammen- und Fortklang alles Menschlichen" (MA II, 186) zu erhalten und, Schritt für Schritt, eine Synthese zu erreichen. Die universale anthropologische Komparatistik, die dafür herangezogen wird, weist historiographische, ethnographische und biographische Momente auf. Es geht um die Rettung des Menschlichen und deswegen um eine Abwendung von aller Philosophie, die, anstatt das Leben voll anzunehmen, es abstrakt und einseitig verkürzt.

Die Forderung nach einer neuen, anthropologisch belehrten Philosophie stellt Nietzsche in einer Situation, in der die Menschheit zum erstenmal in der Geschichte befähigt ist, über die Gestaltung ihrer selbst und der Welt im ganzen auf Gedeih und Verderb $\mathrm{zu}$ entscheiden. Die unumgängliche welthistorische Praxis hat sich vor einer bisher nie dagewesenen umfänglichen Rücksicht auf die Wirklichkeit alles Menschlichen zu verantworten. Insofern Moral, Religion, Philosophie und die damit zusammenhängende Wissenschaft eine umfängliche Wahrung und Förderung alles Menschlichen nicht zulassen und stattdessen die Orientierung an einer Teleologie hochhalten, die vorgibt, die Zwecke der Welt und des Menschen und ein Ziel alles Geschehens zu kennen, lehnt Nietzsche sie $a b$ und kämpft gegen sie. In ihrer Einseitigkeit und Beschränktheit, einmal universal statuiert, würden sie die Verödung und den Untergang des Menschen heraufführen. Angesichts der Ge-

26. Vgl. zu diésem Aphorismus „Eine Art Kultus der Leidenschaften“ in $\mathrm{Z} \mathrm{I}$ : „Von den Freuden- und Leidenschaften" (S II, 302f). 
fahr eines aktiven und totalen Nihilismus setzt Nietzsche auf eine neue Gerechtigkeit: eine umfängliche Einsicht, die zum Vorteil nicht bloß beschränkter Gruppen, sondern aller Menschen zu wirken vermag, indem sie einerseits das Erlebte der ganzen Menschheit zusammendenkt und aus der Geschichte ein Fazit zieht und andererseits durch Überwindung der alten moralischen Werte neu die unerschöpfliche menschliche Natur, damit aber höchstmögliche Differenzierung anstelle von Uniformierung und Nivellierung zur Geltung bringt.

Worauf Nietzsche besteht, ist folgendes: Die Menschen sind nicht einem allgemein aufweisbaren System von Daseinszwecken verantwortlich. Nicht jedem steht jegliche Menschenmöglichkeit zur persönlichen Verwirklichung offen. Für die menschliche Person ist vielmehr von den Prinzipien der Einmaligkeit, Unabänderlichkeit und Unantastbarkeit auszugehen. Erst die Selbstschätzung, die Lust an sich und die Freude am eigenen Tun erlauben auch die Anerkennung der fremden Natur. Dies ist die Voraussetzung für eine Toleranz, die auch noch hinauszuführen vermag über die relative Freiheit und den Partikularismus der freien Geister, der Immoralisten, der artistischen, der viatorischen, der vitalistischen und der solitären Typen. Im Übersteigen dieser Vorformen synthetischer Menschlichkeit und in der Beteiligung an der unerschöpflichen Entfaltung des Menschlichen wird schließlich das Entscheidende in der praktischen Überwindung der Nihilismus-Krise erreicht: die Verwandlung des Ekels am Menschen in die Lust des Menschen am Menschen.

Auf den vollen Geschmack zu kommen, um von der Verleumdung des Lebens abzulassen, am Dasein Freude zu erlangen und in eine gesteigerte Zukunft hineinzufinden - dieses Praktische ist es, was Nietzsche der Philosophie als Aufgabe vorgezeichnet hat. 